OPEN ACCESS

Edited by: Bertrand Kaeffer,

l'alimentation et l'environnement (INRAE), France

Reviewed by:

Sankar Renu,

Upkara Inc., United States

Tarek A. Ahmad,

Bibliotheca Alexandrina, Egypt

${ }^{*}$ Correspondence:

Xiaolu Xiong

xiongxiaolu624@sohu.com

Wenhui Yang

fionyoung@163.com

${ }^{+}$These authors have contributed equally to this work

Specialty section:

This article was submitted to Vaccines and Molecular Therapeutics,

a section of the journal

Frontiers in Immunology

Received: 12 October 2021 Accepted: 05 January 2022 Published: 26 January 2022

Citation:

Zhang W, Song X, Zhai L, Guo J,

Zheng $X$, Zhang L, Lv M, Hu L,

Zhou $D$, Xiong $X$ and Yang $W$

(2022) Complete Protection Against

Yersinia pestis in BALB/c Mouse

Model Elicited by Immunization With

Inhalable Formulations of $r F 1-V 10$

Fusion Protein via Aerosolized

Intratracheal Inoculation.

Front. Immunol. 13:793382.

doi: 10.3389/fimmu.2022.793382

\section{Complete Protection Against Yersinia pestis in BALB/c Mouse Model Elicited by Immunization With Inhalable Formulations of rF1-V10 Fusion Protein via Aerosolized Intratracheal Inoculation}

Wei Zhang ${ }^{\dagger}$, Xiaolin Song ${ }^{\dagger}$, Lina Zhai, Jianshu Guo, Xinying Zheng, Lili Zhang, Meng Lv, Lingfei Hu, Dongsheng Zhou, Xiaolu Xiong * and Wenhui Yang *

State Key Laboratory of Pathogen and Biosecurity, Beijing Institute of Microbiology and Epidemiology, Beijing, China

Pneumonic plague, caused by Yersinia pestis, is an infectious disease with high mortality rates unless treated early with antibiotics. Currently, no FDA-approved vaccine against plague is available for human use. The capsular antigen $\mathrm{F}$ 1, the low-calcium-response $\mathrm{V}$ antigen (LCrV), and the recombinant fusion protein ( $r F 1-L C r V)$ of $Y$. pestis are leading subunit vaccine candidates under intense investigation; however, the inability of recombinant antigens to provide complete protection against pneumonic plague in animal models remains a significant concern. In this study, we compared immunoprotection against pneumonic plague provided by $r F 1, r V 10$ (a truncation of LcrV), and rF1-V10, and vaccinations delivered via aerosolized intratracheal (i.t.) inoculation or subcutaneous (s.c.) injection. We further considered three vaccine formulations: conventional liquid, dry powder produced by spray freeze drying, or dry powder reconstituted in PBS. The main findings are: (i) rF1-V10 immunization with any formulation via i.t. or s.c. routes conferred $100 \%$ protection against $Y$. pestis i.t. infection; (ii) $\mathrm{rF1}$ or $\mathrm{rV} 10$ immunization using i.t. delivery provided significantly stronger protection than rF1 or rV10 immunization via s.c. delivery; and (iii) powder formulations of subunit vaccines induced immune responses and provided protection equivalent to those elicited by unprocessed liquid formulations of vaccines. Our data indicate that immunization with a powder formulation of $\mathrm{rF} 1-\mathrm{V} 10$ vaccines via an i.t. route may be a promising vaccination strategy for providing protective immunity against pneumonic plague.

Keywords: Yersinia pestis, pneumonic plague, subunit vaccine, rF1-V10, dry powder formulation, aerosolized intratracheal inoculation, mucosal immune response 


\section{INTRODUCTION}

Yersinia pestis, a non-motile, facultative intracellular, gram-negative bacterium, is the causative agent of plague (1-3). Plague, a devastating zoonotic disease prevalent in many parts of the world, is transmitted through infected fleas from rodent reservoirs to humans $(4,5)$. It is estimated to have claimed over 200 million human lives during the course of three major human pandemics (6). The most recent outbreak in Madagascar (2017-2018) resulted in $\sim 2400$ cases and $\sim 200$ deaths, raising global concerns (4).The control of human plague outbreaks relies mainly on the rapid confirmation of the diagnosis, isolation and treatment of confirmed and suspected cases (5). For confirmed patients, effective antibiotics (such as tetracycline, streptomycin, chloramphenicol) and preventive therapies must be administered within 24 hours of the onset of symptoms (7). Especially, pneumonic plague transmitting through aerosol droplets is the most dangerous among the three primary clinical forms due to its rapid onset and progression $(6,8)$. Without the rapid response with appropriate antibiotics, the fatality rate of pneumonic plague approaches 100\% (9). Moreover, Y. pestis remains listed as a Tier 1 Select Agent because of its potential use as a biological weapon in an aerosolized form, making it an urgent public health and safety priority $(10,11)$. Therefore, development of a protective vaccine that provides both rapid and long-lasting immunity in the event of mass exposure to aerosolized $Y$. pestis is of great interest.

Historically, killed whole-cell vaccines (KWCVs) and live whole-cell vaccines (LWCVs) have been successfully used to protect humans against plague in parts of the world (12). To prepare KWCVs, Y. pestis were inactivated by heating or with chemicals. These vaccines evoked immunity against bubonic plague but were inefficient against pneumonic plague in animal models $(13,14)$. KWCVs are no longer used due to questionable efficacy and considerable reactogenicity. LWCVs were prepared from fully virulent strains of $Y$. pestis after multiple passages. The former Soviet Union and other nations still use LWCVs for human vaccination, e.g., the NIIEG line of the pgm-negative strain EV76. LWCVs are able to protect humans against bubonic and pneumonic plague (15-17), however, these vaccines are associated with several adverse effects, and fail to provide longterm immunity $(1,18)$. In addition, safety concerns have limited enthusiasm for the development of LWCVs and they are only recommended in endemic areas (19).

The subunit vaccine provides the most promise as a plague vaccine. Development efforts for an effective subunit vaccine to pneumonic plague have focused on two primary antigens of $Y$. pestis, namely the capsular protein (F1) and the low calcium response protein (LcrV). Baker et al. (20) first purified F1 protein and demonstrated that a vaccine with $\mathrm{F} 1$ protected mice and rats from bubonic plague. However, the F1 vaccine candidate provided only $65-84 \%$ protection against pneumonic plague (21); vaccines based exclusively on F1 were ineffective against F1-negative $Y$. pestis, which may be as virulent as wild-type (WT) CO92 Y. pestis (22). Burrows (23) discovered that LcrV was an important virulence protein and subsequent studies confirmed it was a critical protective antigen against $Y$. pestis infection $(19,24,25)$. Unfortunately, part of the LcrV protein, acid residues 271-300, partially suppresses host immune response by stimulating interleukin-10 (IL-10), which suppresses Th1 cells $(26,27)$; this limits its usefulness in vaccines. The combination of recombinant F1 and LcrV antigens (rF1-LcrV) has a good safety profile in various animal models $(28,29)$, elicits greater protection than either F1 or LcrV alone $(30,31)$, but rF1-LcrV does not confer complete protection for mice challenged with more than $255 \times$ $\mathrm{LD}_{50} Y$. pestis administered via inhalation (32-34). Most recently, plague vaccines based on the expression of protective antigens of $Y$. pestis in live vectors (bacterial or viral platform) were developed (35) but had obvious limitations. There is thus a need to continue research on subunit vaccine candidates, which require further modification to minimize shortcomings and elicit more robust immune protection against pneumonic plague.

Over the past few decades, pulmonary delivery of vaccines has received increasing attention due to its ability to recruit local immune responses of the bronchopulmonary mucosa in addition to the broader systemic immune response (36-38). In addition, administration of vaccines via the lungs shows better bioavailability and more rapid effectiveness than injection routes because of the lung's large surface area, abundant blood flow, and highly permeable epithelium $(39,40)$. Currently, there are two formulations of inhalable vaccines: (i) liquid formulations that require a cold chain transport system to maintain vaccine potency; and (ii) powder formulations that have long-term stability at room temperature for storage and shipping $(41,42)$. Given its obvious advantages, the latter is attracting more attention for use in aerosolized intratracheal (i.t.) delivery of vaccines. For more than 70 years, the $Y$. pestis EV NIIEG strain has been used as a human plague vaccine in the former Soviet Union and confers protection against bubonic and pneumonic plague after administration via inhalation $(16,43,44)$. However, the protection appears to be short-lived and the vaccine is highly reactogenic, limiting licensing of this vaccine for use in many parts of the world $(1,12,45)$. The preparation of live $Y$. pestis dry powder is rarely reported in the literature, possibly because of bacterial viability being lost during preparation. Subunit vaccine candidates may thus prove a better option for inhalable powder. In this study, we improve the immunoprotection of subunit vaccines against pneumonic plague by preparing the $\mathrm{rF} 1, \mathrm{rV} 10$ (a truncation of LcrV), or $\mathrm{rF} 1-\mathrm{V} 10$ fusion protein using spray freeze drying (SFD) to generate dry powder with the adjuvant $\mathrm{CpG}$ for i.t. inoculation. We then explore the immunogenicity and protective efficacy of these three subunit vaccines in different formulations (liquid, powder and reconstituted powder) via i.t. and subcutaneous (s.c.) administration routes in a mouse model of $Y$. pestis i.t. infection. Our results demonstrate preclinical feasibility of using a powder formulation of $\mathrm{rF} 1-\mathrm{V} 10$ and the potential use of an alternative pulmonary delivery method.

\section{MATERIALS AND METHODS}

\subsection{Expression and Purification of $\mathrm{rF1}$, rV10, and rF1-V10}

Recombinant plasmids were built as shown in Figure 1. The primers used for DNA synthesis are listed in Supplementary Table 1. 
The DNA sequence encoding $\mathrm{rF} 1\left(\mathrm{~F}_{22-170}\right.$, Accession No.: NC_003134) without signal peptide (46) was amplified by PCR. Previous studies have reported that rV10, lacking amino acid residues 271 to $300\left(\mathrm{LcrV}_{271-300}\right)$, lost the ability to induce IL-10, significantly reducing its immunosuppressive properties on mice compared to the intact $\operatorname{LcrV}(27,47)$. Therefore, in our study, the sequence encoding the immunosuppressive fragment of LcrV (LcrV 271-300, Accession No.: NC_003131) was removed and the DNA sequences encoding $\mathrm{LcrV}_{1-270}$, the linker Gly-Thr dipeptide, and $\mathrm{LcrV}_{301-326}$ were fused in sequential order to obtain rV10 by overlap extension PCR. In brief, the DNA fragments of $\mathrm{LcrV}_{1-270}$ and $\mathrm{LcrV}_{301-326}$ were generated using primer pairs (rV10-F0, rV10-R0) and (rV10-F, rV10-R; these contain the DNA sequence of the linker) in the first PCR; these two DNA fragments were subsequently used as templates in the second PCR with rV10-F0 and rV10-R0 primers to generate rV10. To obtain the rF1-V10 sequence, DNA sequences encoding $\mathrm{rF} 1$, the linker Glu-Phe dipeptide, and rV10 were fused in sequential order by overlap extension PCR, and the two PCR products ( $\mathrm{rF1}$ and $\mathrm{rV} 10$ ) generated using primer pairs (rF1-F0 and rV10-R0) and (rF1-V10-F and rF1-V10-R; these contain the DNA sequence of the linker) in the first PCR.
The $\mathrm{rF1}-\mathrm{V} 10$ were generated by a second PCR using primer pairs rF1-F0 and rV10-R0. Finally, the DNA fragment rF1, rV10, or $\mathrm{rF1-V10}$ was inserted into the BamHI/XhoI digested pSMART-I (Ming Chen Zhi Yuan Biotechnology Co. LTD., Beijing, China) plasmid by In-Fusion cloning to generate pSMART-rF1, pSMART-rV10, or pSMART-rF1-V10 vectors, respectively.

These three recombinant proteins were purified by affinity chromatography from lysates of E. coli BL21 cells (Novagen, Madison, WI, USA) following a previously published protocol (27). The His-Sumo tags at the $\mathrm{N}$ terminus of the purified proteins were excised by Sumo Protease (ULP403) and the resulting proteins were analyzed by SDS-PAGE and Western blot. Protein concentration was analyzed using the bicinchoninic acid (BCA) method (Pierce Biotechnology; Rockford; IL). Endotoxin level was assayed using Limulus amebocyte lysate (QCL-1000; Cambrex, NJ) and found to be 10 Endotoxin Units (EU) per $1 \mathrm{mg}$ purified protein (48).

\subsection{Preparation of Powder Formulations of the rF1, rV10, and rF1-V10 Vaccines}

The subunit vaccine powders were prepared by SFD as described in published literature $(49,50)$. Specifically, each subunit vaccine

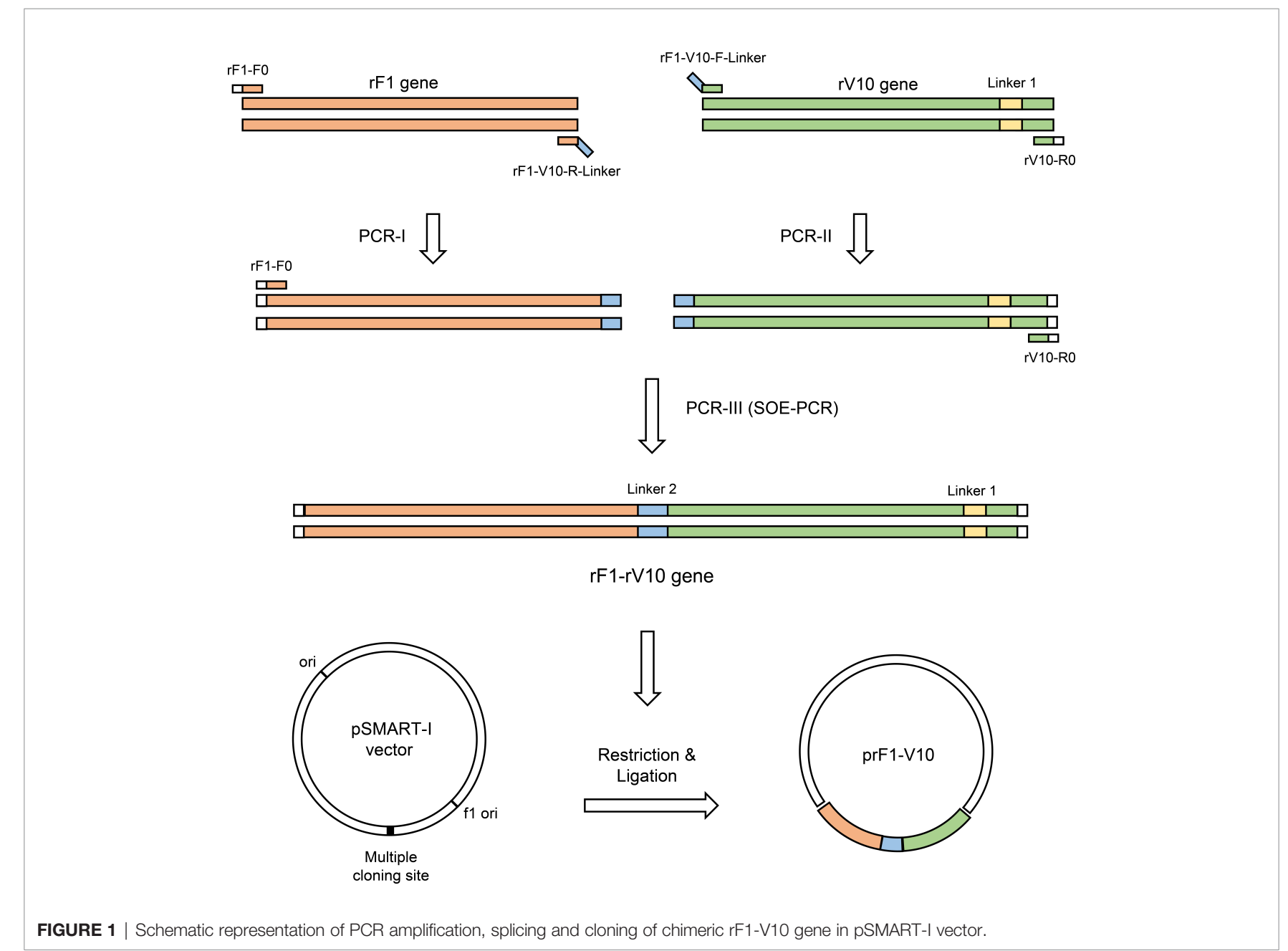


was dissolved in spray drying solution containing D-mannitol, myo-inositol, L-leucine, and poloxamer 188 as excipients and CpG as a mucosal adjuvant (51). This solution was kept in an ice bath and then sprayed into a vessel of liquid nitrogen with a twofluid pneumatic spray nozzle $(2 \mathrm{~mm}$ diameter; TSE Systems $\mathrm{GmbH}$, Berlin, Germany) using a fixed back pressure of 1.5 bar and a liquid feed rate of $5 \mathrm{ml} / \mathrm{min}$. The sprayed atomized droplets were quickly frozen into ice crystals under liquid nitrogen. After liquid nitrogen was evaporated, the ice crystals were transferred to a stainless-steel vessel followed by lyophilization in a vacuum freeze-drying system for $48 \mathrm{~h}$ at a manifold temperature of $-55^{\circ} \mathrm{C}$ and a vacuum pressure of $10 \mathrm{~Pa}$. The obtained powders were stored at $4^{\circ} \mathrm{C}$ until further use.

To evaluate the quality and stability of the dry powder formulation, these subunit vaccines were reconstituted in deionized water, then analyzed by SDS-PAGE and Western blot (using sera collected from immunized mice). The volume median diameter (VMD) of dry powder vaccines was measured using a laser particle size analyzer (RODOS \& HELOS, Sympatec, ClausthalZellerfeld, Germany) and the mass median aerodynamic diameter (MMAD) of the vaccine aerosol particles was measured with the aerodynamic particle sizer (APS) spectrometer 3321 (52) (TSI Inc., St. Paul, MN). The moisture content of vaccine dry powders was determined using thermogravimetric analysis (TGA), as described previously (50). Particle morphology was examined with a Hitachi S-3400N scanning electron microscope.

\subsection{Animals}

Female BALB/c mice (SPF) at 6-weeks of age were obtained from Charles River Laboratories (Beijing, China). This study was approved by the Institute of Animal Care and Use Committee (IACUC) at the Academy of Military Medical Sciences (AMMS), ethical approval number IACUC-DWZX-2021-057.

\subsection{Bacteria Strain and Growth Media}

$Y$. pestis strain 201, which is avirulent to humans (53), is maintained in our laboratory. It was cultivated in Brain Heart Infusion broth (BHI; BD, Voigt Global Distribution Inc., Lawrence, KS). Cultures were inoculated overnight with $\mathrm{BHI}$ (dilution $1: 20$ ) and cultured at $26^{\circ} \mathrm{C}$ in a shaking incubator at 220 $\mathrm{rpm}$ to an optical density at $600 \mathrm{~nm}$ (OD600) of $\sim 1.0$. Cultures were then inoculated with $\mathrm{BHI}$ (dilution 1:100) and maintained at $26^{\circ} \mathrm{C}$, again to $\mathrm{OD} 600 \sim 1.0$. After that, cultures were transferred to a $37^{\circ} \mathrm{C}$ shaking incubator for another $3 \mathrm{~h}$. For growth on a solid surface, $Y$. pestis was grown on $5 \%$ sheep blood agar (SBA) plates (Luqiao, Beijing, China) at $26^{\circ} \mathrm{C}$ for 3 days.

\section{$2.5 \mathrm{Immunization}$ and Challenge}

Mice (41 per group) were immunized three times at 3-week intervals (days 0,21 , and 42 ) through i.t. or s.c. routes (Figure 2). The i.t. immunization was performed using a Dry Powder Insufflator for powder inoculation and a MicroSprayer Aerosolizer for reconstituted powder or liquid inoculation following previously described methods (54). Group details are shown in Table $\mathbf{1}$ (five experimental groups per protein, five negative controls, and two blank controls). For rF1, the five experimental groups included three groups of mice i.t. inoculated with 1) $0.5 \mathrm{mg}$ of $\mathrm{rF} 1$ dry powder (i.t.-rF1, powder), 2) $0.5 \mathrm{mg}$ of $\mathrm{rF} 1$ dry powder reconstituted in PBS (i.t.-rF1, reconstituted powder) to create a reconstituted solution, designed as a quality control to assess the influence of the SFD process on vaccine efficacy, and 3) $20 \mu \mathrm{g}$ of $\mathrm{rF1}$ liquid without SFD (i.t.-rF1, liquid) in PBS. Since the powder vaccine is not suitable for use in s.c. injection, the next two groups of mice were inoculated via s.c. with 4) $0.5 \mathrm{mg} \mathrm{rF} 1$ dry powder reconstituted in PBS (s.c.-rF1, reconstituted powder), or 5) $20 \mu \mathrm{g}$ of $\mathrm{rF} 1$ liquid without SFD (s.c.-rF1, liquid). The same five experimental group treatments were used for $\mathrm{rV} 10$ and $\mathrm{rF1-V10}$ vaccines. Mice in negative controls were immunized with $\mathrm{CpG}$ and those in blank controls were immunized with PBS.

At 63 days post-primary immunization (dppi), immunized mice were anesthetized by intraperitoneal injection of pentobarbital sodium and then challenged with $50 \times \mathrm{LD}_{50}$ $(1,000 \mathrm{CFU})$ or $1,000 \times \mathrm{LD}_{50}(20,000 \mathrm{CFU})$ of $Y$. pestis strain 201 through an i.t. route using a MicroSprayer Aerosolizer. The direct inhalation of aerosolized $Y$. pestis was used to better mimic the natural aerosol infection of pneumonic plague. Animals were checked daily for morbidity and mortality over the course of 2 weeks. At days 2, 7, and 14 post-challenge, bacterial load analysis was done for the low-dose challenge groups, three mice per group were sacrificed. The lungs, spleens, and livers of these mice were harvested individually and approximately $100 \mathrm{mg}$ of each

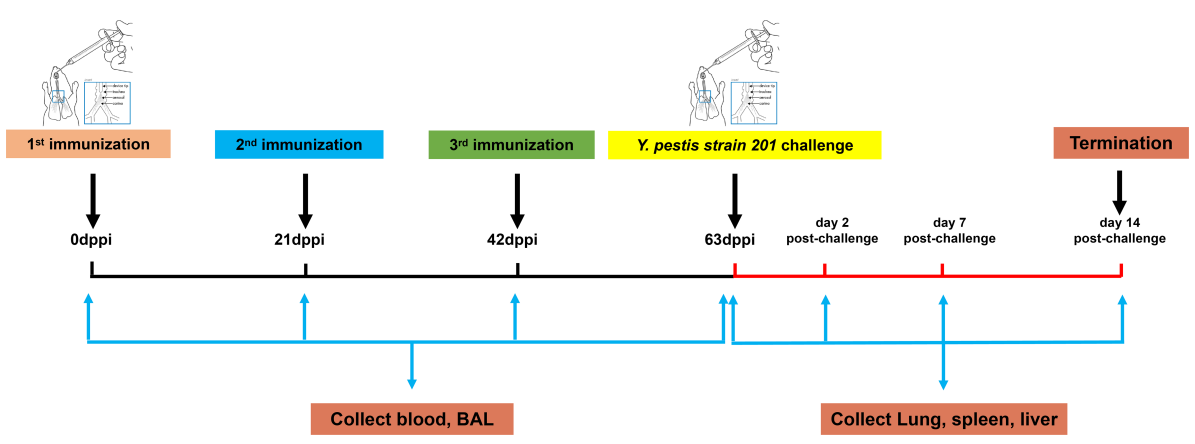

FIGURE 2 | Schema of the immunization protocol. BAL, bronchoalveolar lavage; dppi, days post-primary immunization. 
TABLE 1 | Summary of immunization groups used in the experiment.

\begin{tabular}{|c|c|c|c|c|c|c|}
\hline Number & Group & Immunization route & Formulation type & Antigen dose ( $\mu \mathrm{g} / \mathrm{mouse})$ & CpG dose ( $\mu \mathrm{g} / \mathrm{mouse})$ & Volume ( $\mu \mathrm{l} /$ mouse) \\
\hline 1 & rF1 & i.t. & powder & 20 & 20 & 50 \\
\hline 2 & & & powder reconstituted & 20 & 20 & 50 \\
\hline 3 & & & liquid & 20 & 20 & 50 \\
\hline 4 & & s.c. & powder reconstituted & 20 & 20 & 100 \\
\hline 5 & & & liquid & 20 & 20 & 100 \\
\hline 6 & rV10 & i.t. & powder & 20 & 20 & 50 \\
\hline 7 & & & powder reconstituted & 20 & 20 & 50 \\
\hline 8 & & & liquid & 20 & 20 & 50 \\
\hline 9 & & s.c. & powder reconstituted & 20 & 20 & 100 \\
\hline 10 & & & liquid & 20 & 20 & 100 \\
\hline 11 & $\mathrm{rF} 1-\mathrm{V} 10$ & i.t. & powder & 20 & 20 & 50 \\
\hline 12 & & & powder reconstituted & 20 & 20 & 50 \\
\hline 13 & & & liquid & 20 & 20 & 50 \\
\hline 14 & & s.c. & powder reconstituted & 20 & 20 & 100 \\
\hline 15 & & & liquid & 20 & 20 & 100 \\
\hline 16 & CpG & i.t. & powder & / & 20 & 50 \\
\hline 17 & & & powder reconstituted & / & 20 & 50 \\
\hline 18 & & & liquid & / & 20 & 50 \\
\hline 19 & & s.c. & powder reconstituted & / & 20 & 100 \\
\hline 20 & & & liquid & / & 20 & 100 \\
\hline 21 & PBS & i.t. & liquid & / & / & 50 \\
\hline 22 & & S.c. & liquid & / & / & 100 \\
\hline
\end{tabular}

i.t., aerosolized intratracheal inoculation; s.c., subcutaneous injection.

organ homogenized in $800 \mu \mathrm{l}$ PBS. Samples of $50 \mu \mathrm{l}$ of homogenate were diluted serially in PBS, and $10 \mu \mathrm{l}$ dilutions were plated onto blood agar plates for bacterial counting as previously described (54).

\subsection{ELISA Assay of Specific Antibody}

Sera and bronchoalveolar lavage (BAL) from four mice per group were collected after each immunization (at 21, 42 and 63 dppi). The titers of IgG (sera and BAL) and IgA (BAL) antibodies against the cognate recombinant proteins were evaluated by enzyme-linked immunosorbent assay (ELISA) as described in previous studies (55). Briefly, individual wells in 96-well plates were coated with $\mathrm{rF} 1, \mathrm{rV} 10$, or $\mathrm{rF1}$-V10, plates were blocked with bovine serum albumin (BSA) and incubated with serially diluted sera collected from cognate antigen immunized mice. After washing, the plates were incubated with HRP-conjugated goat anti-mouse IgG or IgA (Abcam, Cambridge, MA). 3,3',5,5'tetramethylbenzedine (TMB) was used as substrate, and optical density (OD) was measured at $450 \mathrm{~nm}$ with a reference filter $(630 \mathrm{~nm})$.

\subsection{ELISPOT Assay}

At $63 \mathrm{dppi}$, total mononuclear cells were isolated from spleens of three mice per group and suspended $\left(1 \times 10^{7} \cdot \mathrm{ml}^{-1}\right)$ in DMEM basic medium (Gibco, Shanghai, China) containing 10\% (v/v) fetal bovine serum (Gibco, Australia) and 1\% (v/v) penicillinstreptomycin (Gibco, Grand Island, USA). The cells were then plated into a 96-well ELISPOT plate (Mabtech, Nacka Strand, Sweden) with $1 \times 10^{6}$ cells per well, as previously described. The cells in each well were stimulated with $5 \mu \mathrm{g}$ of each cognate protein $(\mathrm{rF} 1, \mathrm{rV} 10$, or $\mathrm{rF} 1-\mathrm{V} 10)$, Concanavalin A (ConA, positive control, Sigma-Aldrich, Germany), or cell culture medium (negative control) and then incubated for $20 \mathrm{~h}$ at $37^{\circ} \mathrm{C}$ under $5 \% \mathrm{CO}_{2}$.
The levels of IFN- $\gamma$ or IL-4 were measured by ELISPOT assays as previously described (56).

\subsection{Histopathology}

Animal tissues from three mice per group were collected at 63 dppi and day 2 post-challenge, fixed in $4 \%$ paraformaldehyde and embedded in paraffin. Blocks were cut into $5-\mu \mathrm{m}$ sections, which were stained with hematoxylin and eosin (HE). Pathological alterations in tissue slices were observed by light microscopy. Tissue sections were evaluated blind by a trained pathologist according to the following scores: 0 , no pathological lesions; 1 , minimal; 2 , mild; 3 , moderate; 4 , severe.

\subsection{Statistical Analysis}

Data are expressed as mean \pm SEM. All statistical analyses were performed using SAS statistical software (version 9.1, SAS Institute Inc., Cary, NC) or GraphPad Prism 8.0. Differences in the levels of antibodies and bacterial load among all groups of mice were tested using a two-way ANOVA, followed by LSD analysis or Tukey's test. IFN- $\gamma$ and IL-4 levels were compared by one-way ANOVA, followed by LSD analysis. Mouse survival rate was analyzed using Kaplan-Meier survival estimates. $P<0.05$ was considered significantly different for all statistical analyses.

\section{RESULTS}

\subsection{Preparation and Characterization of Pulmonary Delivery Vaccines}

Following preparation, dry powder vaccines were characterized for structural integrity, immunogenicity, morphology, uniformity of distribution, residual moisture content, and aerodynamic parameters. Molecular weights of reconstituted $\mathrm{rF} 1, \mathrm{~V} 10$, and 
rF1-V10 powders were identical to those of the liquid formulation $(\sim 16, \sim 34$, and $\sim 50 \mathrm{kDa}$, respectively; Figure 3A). Both reconstituted powder and the liquid formulations reacted to sera from cognate antigen immunized mice, and titers did not differ significantly (Figures 3A, B). The results demonstrate that biochemical integrity and immunogenicity of the three vaccines were unaffected by the SFD process.

Scanning electron micrographs (SEM) of the three vaccine dry powders ( $\mathrm{rF1} 1-\mathrm{V} 10, \mathrm{rV10}$, and $\mathrm{rF1}$ ) are shown in Figure 3C. As expected, the particles were spherical and porous. Three random areas in an SEM graph of the dry powder were chosen for analysis. The VMD of the three vaccine powders, as determined by a laser particle size analyzer, were $7.85 \pm 0.68 \mu \mathrm{m}(\mathrm{rF} 1), 9.75 \pm 0.33 \mu \mathrm{m}$ (rV10), and 13.16 $\pm 0.27 \mu \mathrm{m}(\mathrm{rF1}-\mathrm{V} 10)$ (Figure 3D). Moreover, the MMAD of aerosol particles, as measured by APS spectrometer 3321, were $2.45 \pm 0.05 \mu \mathrm{m}(\mathrm{rF} 1), 2.45 \pm 0.09 \mu \mathrm{m}(\mathrm{rV} 10)$, and $2.17 \pm$ $0.06 \mu \mathrm{m}(\mathrm{rF1}-\mathrm{V} 10)$ (Figure 3E). The residual moisture content of the vaccine dry powders was determined by TGA to be approximately $0.971 \%(\mathrm{rF} 1), 0.678 \%(\mathrm{rV} 10)$, and $0.653 \%(\mathrm{rF} 1-$ V10) (w/w) (Figure 3F). The results indicate that all three vaccine dry powders prepared were suitable for aerosol inhalation.

\subsection{Systemic Humoral and Lung Mucosal Immune Responses Induced by rF1, rV10 and $\mathrm{rF} 1-\mathrm{V} 10$ Vaccines via i.t.}

Antigen-specific IgG in sera and antigen-specific IgG and secretory immunoglobulin A (SIgA) in BAL were evaluated after delivery of the three subunit vaccines ( $\mathrm{rF} 1, \mathrm{rV} 10$ and $\mathrm{rF} 1$ V10) with different formulations (liquid, powder, and reconstituted powder) and via different routes (i.t. and s.c.). The antibodies against native F1 or LcrV were not tested in this study, because extractions of these proteins require a laborious and time-consuming processes and result in low efficiency and purity.

\subsubsection{Systemic Humoral Immune Responses Induced by Vaccines via i.t.}

A substantial and progressive induction of antigen-specific antibody was observed in all groups (Figure 4). Booster vaccinations with the same formulation used in the primary vaccine resulted in significant increases in antibody levels at 42 dppi and 63 dppi. At 63 dppi, in rF1-V10-immunized groups, levels of specific IgG in the serum of mice immunized via i.t.-rF1$\mathrm{V} 10$ were similar to those for mice immunized via s.c.-rF1-V10 (Figure 4A). In rF1- and rV10-immunized groups, the levels of IgG in serum of mice immunized via i.t.-rF1 or i.t.-rV10 were significantly higher than those in mice immunized via s.c.-rF1 or s.c.-rV10 $(P<0.05$, Figures 4 D, G). Moreover, no significant differences in levels of specific IgG were observed among different formulations (dry powder, reconstituted powder, liquid) of each subunit vaccine $(P>0.05)$. These results suggest that immunization with $\mathrm{rF} 1-\mathrm{V} 10$ can induce a strong humoral immune response regardless of the delivery route and formulation used.
A
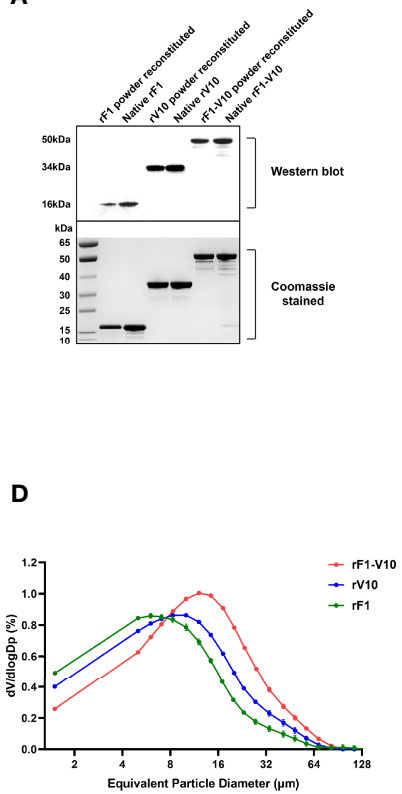

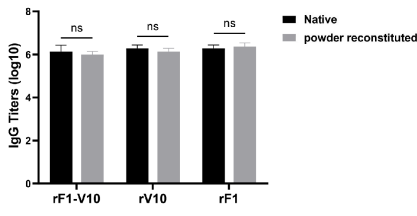

E

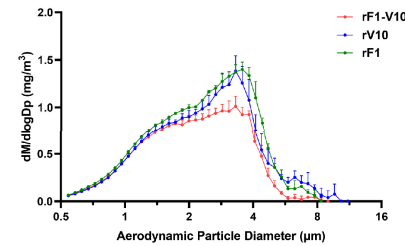

C

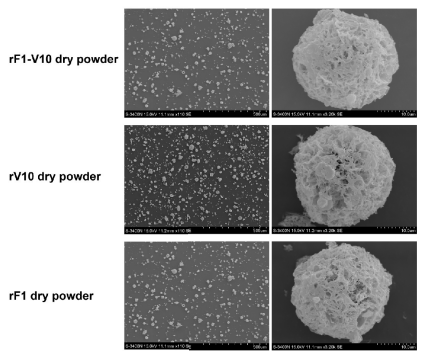

$\mathbf{F}$

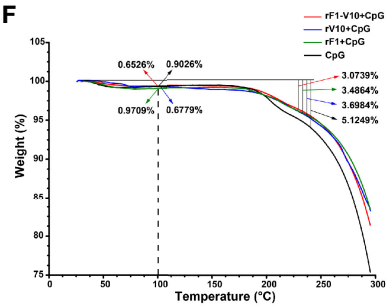

FIGURE 3 | Characterization of rF1, rV10 and rF1-V10 dry powders. (A) Dry powders and liquids of three vaccines were analyzed by Western blot using sera collected from cognate antigen immunized mice (upper panel). A protein gel stained with Coomassie brilliant blue was used as a loading control (lower panel). (B) The immunogenicity of dry powder and liquid vaccines measured by ELISA, using sera collected from cognate antigen immunized mice. (C) Scanning electron microscopy pictures of the three vaccine dry powders. (D) VMD of the aerosolized vaccine dry powders, as determined using a laser particle size analyzer. (E) MMAD of the aerosolized vaccine dry powders, as measured using a TSI APS 3321. (F) TGA of the three vaccine dry powders. ns, no statistical significance. 


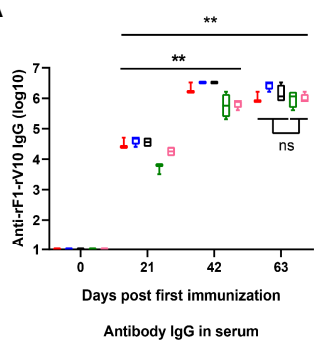

D

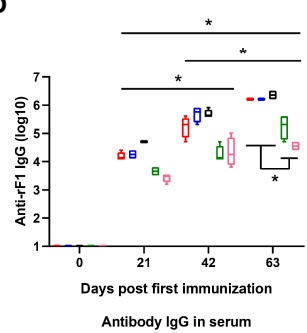

G

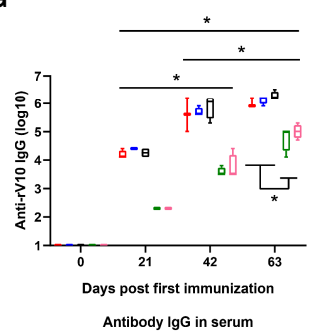

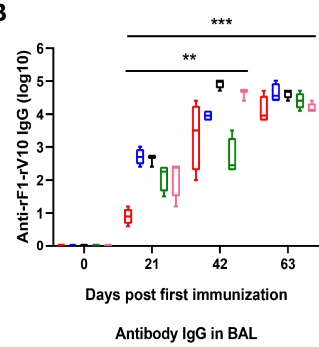

E
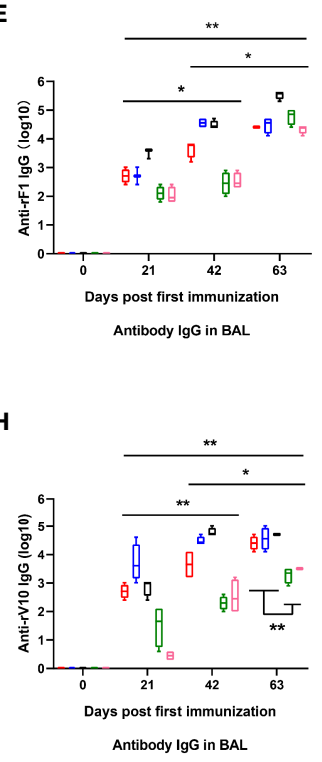

C

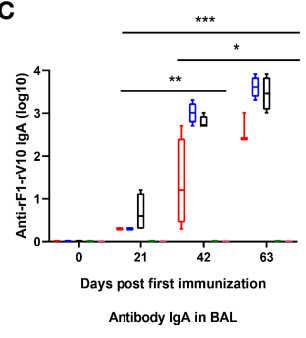

므 it.trF1-rV10 (powder)

ㅁ i.t.-FF1-rV10 (powder reconstituted)

ㅁ i.t.-FF1-rV10 (liquid)

$\square$ s.c.-rF1-rV10 (powder reconstituted)

$\square$ s.c.-rF1-rV10 (liquid)
$\mathbf{F}$

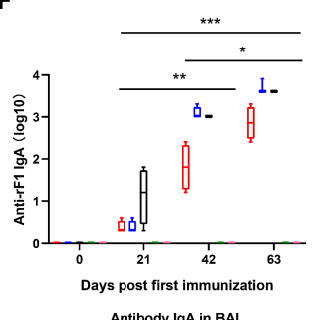

마.t.t. F 1 (powder)

- i.t.-FF1 (powder reconstituted)

ㅁ i.t.rF1 (liquid)

ㅁ s.c.. $\mathrm{F} 1$ (powder reconstituted)

$\square$ s.c. - FF1 (iquid)

FIGURE 4 | Humoral and mucosal antibody responses (mean \pm SD) in mice at 21-, 42- and 63-days post-immunization elicited by rF1-V10, rF1, and rV10 vaccines via i.t. (powder, powder reconstituted and liquid formulations) or s.c. (powder reconstituted and liquid formulations). (A, D, G) Reciprocal serum titters of IgG to (A) rF1-V10, (D) rF1, and (G) rV10. (B, E, H) Reciprocal BAL titers of IgG to (B) rF1-V10, (E) rF1, and (H) rV10. (C, F, I) Reciprocal BAL titers of IgA to (C) rF1-V10, (F) rF1, and (I) rV10. Serum and BAL were collected from four mice per group at 21, 42, and 63 dppi and titers were measured by ELISA. Statistical differences were calculated by two-way ANOVA, followed by least significant difference (LSD) analysis or Tukey's test. ${ }^{*} P<0.05 ;{ }^{* *} P<0.01 ;{ }^{* \star *} P<0.001$.

\subsubsection{Lung Mucosal Immune Responses Induced by Vaccines via i.t}

Since IgG and SIgA both exist in mucosal surfaces (57), the levels of IgG and SIgA in the BAL were tested to evaluate mucosal immune response. The IgG levels in BAL from the i.t.-rF1-V10 and i.t.-rF1 immunized group were comparable to the levels induced in the s.c.-rF1-V10 and s.c.-rF1 immunized group $(P>0.05)$, while i.t.-rV10-immunization elicited a significantly higher antibody response than that generated by s.c.-rV10immunization $(P<0.05$, Figures $\mathbf{4 B}, \mathbf{E}, \mathbf{H})$. The specific SIgA in BAL was not detected in BAL of mice immunized with s.c.-rF1, s.c.-rV10, or s.c.-rF1-V10 (Figures 4C, F, I). Antigen-specific SIgA was detected in BAL after immunization with any of the three formulations in i.t.-immunized mice as early as $21 \mathrm{dppi}$, and levels increased gradually with immunization dose $(P<0.05$, Figures $4 C$, F, I). No significant difference in specific IgA levels in BAL were observed among different vaccine formulations $(P>0.05)$. These results indicate that vaccine immunization via i.t. effectively induces lung mucosal SIgA production.

\subsection{Elevated Cellular Immune Response Elicited by rF1, rV10 and rF1-V10 Vaccines via i.t}

To investigate the specific $\mathrm{T}$ cell-mediated immune response, IFN- $\gamma$ and interleukin (IL)-4 secretion levels were measured. As shown in Supplementary Figure 1, the secretion levels of IFN- $\gamma$ and IL-4 in mice vaccinated via i.t. delivery were significantly higher than those in mice vaccinated via s.c. delivery $(P<0.05)$. No significant difference was observed among different vaccine formulations in i.t.- or s.c.-immunized mice $(P>0.05)$. Vaccineimmunized groups had significantly higher levels of IFN- $\gamma$ and IL-4 compared to control groups $(P<0.05)$.

\subsection{Enhanced Protection Provided by Immunization With rF1-V10 via i.t. or s.c. Following Aerosol Challenge With Y. pestis Strain 201}

In all immunized groups, the protection conferred by reconstituted powder formulations of vaccines was essentially 
equal to that conferred by powder or liquid formulations $(P>$ $0.05)$; thus, for clarity, only powder and liquid formulations are shown in the figures.

\subsubsection{Protection Efficacy After Aerosol Challenge With $50 \times \mathrm{LD}_{50}$ of $Y$. pestis Strain 201}

All mice in PBS (data not shown) and CpG (Figures 5A, C, E) control groups died within 6 days. For rF1-V10 immunized groups, immunization, regardless of the formulation and route of administration, conferred $100 \%$ protection against lethal challenge doses of $Y$. pestis i.t. (Figure 5A). For rF1 immunized groups, the survival rate of mice immunized with powder or liquid (70\% or $80 \%$ survival, respectively) formulations via i.t. was significantly higher than that of mice immunized with liquid (40\% survival) formulation via s.c. $(P<0.05$, Figure 5C). For rV10 immunized groups, mice immunized with powder or liquid formulation via i.t. delivery had significantly higher survival rates (90\% and $100 \%$ survival, respectively) compared to those immunized with liquid formulation via s.c. (40\% survival, $P<$ 0.05 , Figure $5 E$ ). In addition, the survival of mice receiving s.c.-rF1V10 immunization (100\% survival) was significantly higher than that of mice receiving s.c.-rF1 or s.c.-rV10 immunization (both $40 \%$ survival, $P<0.05$, Figures 5A, C, E), while the survival rates of mice receiving i.t.-rF1-V10 immunization and those receiving i.t.-rF1 or i.t.-rV10 immunization were not significantly different $(P>0.05)$.

\subsubsection{Protection Efficacy After Aerosol Challenge With $1,000 \times L_{50}$ of $Y$. pestis Strain 201}

When mice were challenged with a higher dose of $1,000 \times \mathrm{LD}_{50} Y$. pestis strain 201 i.t. at $63 \mathrm{dppi}$, all PBS-immunized mice (data not shown) and CpG-immunized mice (Figures 5B, D, F) died within 4 days. For rF1-immunized groups, the survival percentage of s.c.-rF1-immunized mice ( $0 \%$ survival) was significantly lower than that of i.t.- rF1-immunized mice $(40 \%$ survival in powder group; 50\% survival in liquid group; $P<$ 0.001, Figure 5D), and, for each of these groups, survival was
A

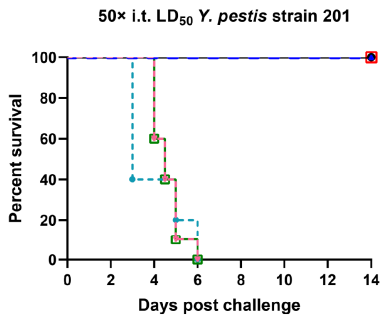

C

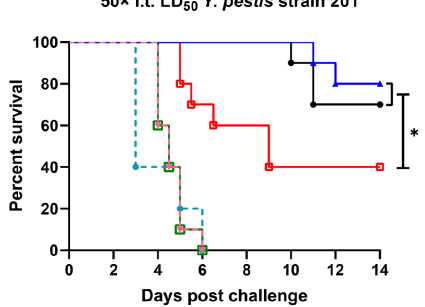

E

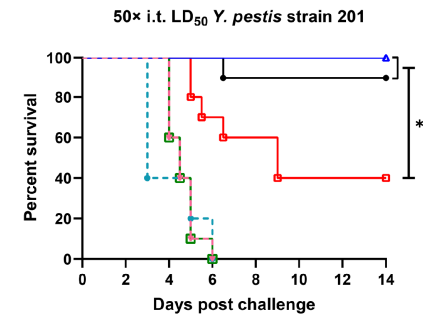

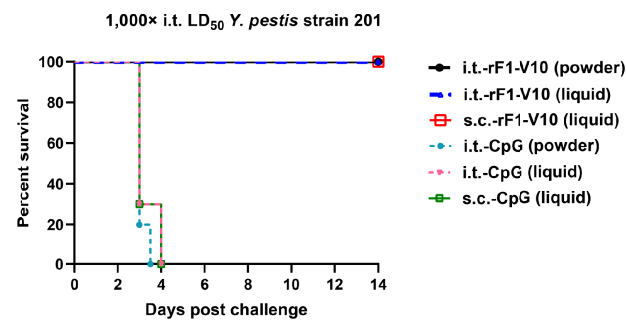

D

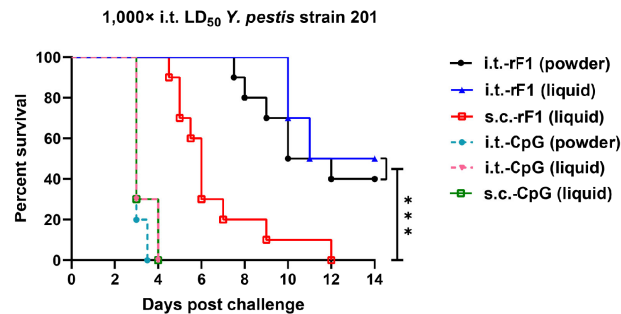

$\mathbf{F}$

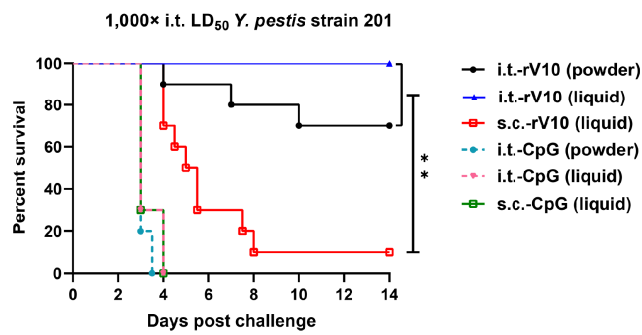

FIGURE 5 | Survival curves for mice challenged with aerosolized Y. pestis strain 201. Mice were immunized three times with $\mathrm{rF1}$, $\mathrm{rV} 10$, or $\mathrm{rF} 1-\mathrm{V} 10$ in one of three formulations (powder; liquid; or powder reconstituted, not shown) via i.t. or s.c. (A, C, E) Mice $(n=10)$ immunized three times with (A) rF1-V10, (C) rF1, or (E) rV10 and then challenged with 50x $L D_{50}$ i.t. at 63 dppi. (B, D, F) Mice $(n=10)$ immunized three times with (B) rF1-V10, (D) $r F 1$, or $(\mathbf{F}) r V 10$ and then challenged with $1,000 \times L_{50}$ i.t. For vaccines, inoculations are shown for powder and liquid formulations (powder reconstituted vaccine data are not shown) administered via i.t. or s.c. routes. Mortality data were analyzed using Kaplan-Meier survival analysis. ${ }^{*} P<0.05 ;{ }^{* *} P<0.01 ;{ }^{* \star *} P<0.001$. 
lower than when challenged with $50 \times \mathrm{LD}_{50} Y$. pestis i.t. $(40 \%$ survival in s.c. groups; $70 \%$ survival in i.t.-powder group; $80 \%$ survival in i.t.-liquid group). For rV10-immunized groups, survival of s.c.-rV10-immunized mice was further reduced to less than $10 \%$, considerably lower than that of i.t.-rV10-immunized ( $>70 \%$ survival) mice $(P<0.01$, Figure 5F). However, i.t.-rV10 immunization with liquid formulation and rF1-V10 immunization (all formulations and routes) still conferred complete protection against $1,000 \times \mathrm{LD}_{50}$ i.t. $Y$. pestis challenge. Overall, these results indicate that $\mathrm{rF1-V10}$ is an excellent subunit vaccine and that the i.t. immunization route is superior to the s.c. immunization route for protection against $Y$. pestis i.t. infection.

\subsection{Bacterial Enumeration From Mouse Organs Following i.t. Challenge}

Bacterial loads in different tissues of vaccine-immunized mice following i.t challenge at $50 \times \mathrm{LD}_{50} Y$. pestis were examined to further evaluate the protection provided by subunit vaccine immunization. At day 2 post-challenge, bacterial loads were below the lowest detectable limit of the assay for lungs, livers, and spleens from i.t.-rF1, s.c.-rF1 and s.c.-rV10-immunized groups; at day 7 post-challenge, bacterial loads ranged from $10^{3} \mathrm{CFU} / \mathrm{g}$ to $10^{7} \mathrm{CFU} / \mathrm{g}$, indicating a mild proliferation of $Y$. pestis in these groups. But by day 14 post-challenge, bacteria had been cleared from tissues (Figures 6D-I). No bacteria were detected at the lowest dilution of organs from rF1-V10immunized mice at days 2, 7 or 14 post-challenges (Figures 6A-C), indicating that immunization with $\mathrm{rF1-V10}$, regardless of route or formulation, had an appreciable protective effect against $Y$. pestis. For control groups, extensive bacteria were found in lungs, spleens, and livers of naive mice at day 2 post-challenge, and all naive mice succumbed to the $Y$. pestis challenge by day 6 post-challenge.

\subsection{Histopathological Analysis of Mouse Tissues After Immunization and Challenge}

The safety of i.t. vaccine immunization was evaluated by examining pathological changes in mouse tissues after vaccination. No obvious pathological lesions were observed in
A

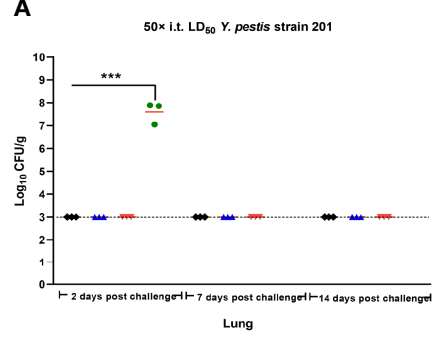

D

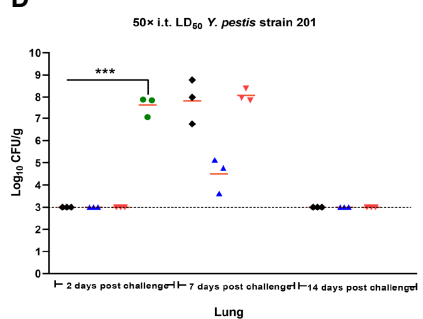

G

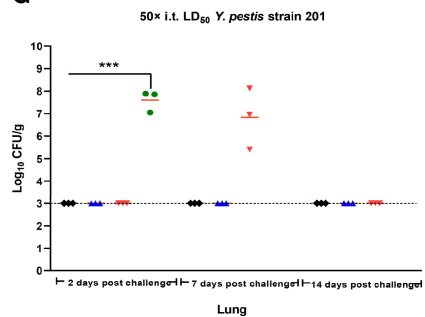

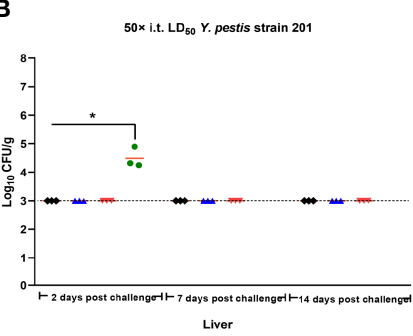

E

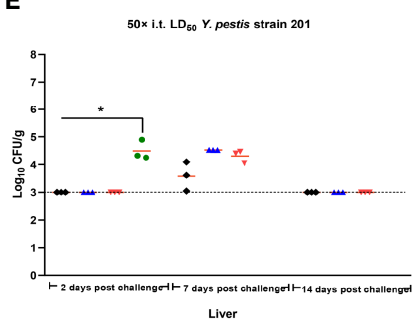

H

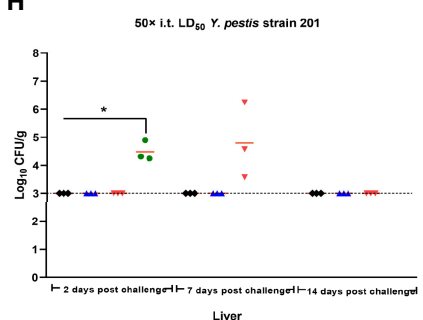

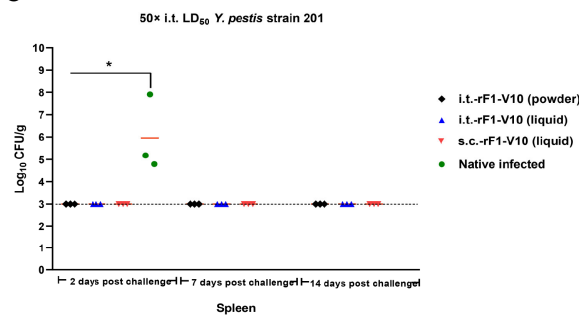

$\mathbf{F}$

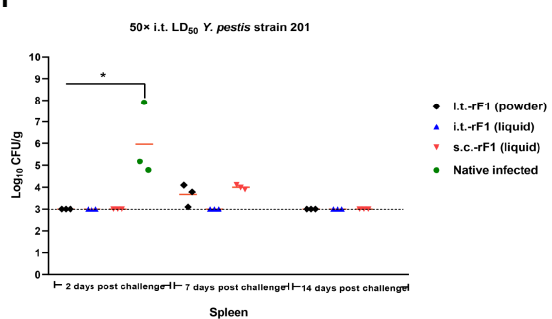

I

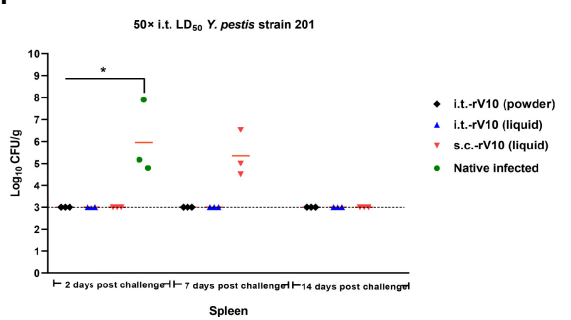

FIGURE 6 | Bacterial load in the organs of mice euthanized at days 2, 7, and 14 post 50x LD 50 of $Y$. pestis strain 201 i.t. challenge. (A-C) Bacterial load in (A) lungs, (B) liver, and (C) spleen of mice immunized with rF1-V10 dry power and liquid formulations. (D-F) Bacterial loads in (D) lungs, (E) liver and (F) spleen of mice immunized with rF1 dry power and liquid formulations. (G-I) Bacterial loads in (G) lungs, (H) liver and (I) spleen of mice immunized with rV10 dry power and liquid formulations. Experiments were performed twice independently with similar results. Data are expressed as the mean $\pm \operatorname{SD}(n=3)$ using data collected from one representative experiment. ${ }^{\star} P<0.05 ;{ }^{\star \star \star} P<0.001$. 
lungs, livers, or spleens of mice immunized with any formulation of vaccines, with $\mathrm{CpG}$, or with $\mathrm{PBS}$ via i.t. or s.c. routes, confirming the safety of i.t. immunization (Supplementary Figures 2A-C).

The efficacy of different vaccines was further assessed by comparing the pathological changes in mouse tissues after $50 \times$ $\mathrm{LD}_{50}$ i.t. challenge. In unvaccinated infected mice, perivascular edema, neutrophil infiltration, and hemorrhage were found in the lungs, and inflammatory cells were recruited to the liver and the white pulp of the spleen (Supplementary Figures 2D-F). No obvious lesions were observed in vaccine-immunized groups, except for the rF1-immunized groups, which had mild inflammation in the lungs. Furthermore, the pathological scores in the lungs, livers, and spleens of the unvaccinated infected control group were significantly higher than those of the vaccine-immunized groups $(P<0.05$, Figure 7$)$.

\section{DISCUSSION}

Due to the sporadic outbreak of pneumonic plague and the threat should it be weaponized, developing effective vaccines against pneumonic plague is important. Compared with F1 or $\mathrm{LcrV}$ alone, a subunit vaccine based on a fusion of F1-LcrV proteins is a promising subunit vaccine $(30,31)$. Previous studies reported that immunization with the $\mathrm{rF1}$-LcrV fusion protein through the s.c. route could fully protect mice only at $10 \times \mathrm{LD}_{50}$ virulent $Y$. pestis CO92 via intranasal (i.n.) challenge (34) and only partially protect mice at $70 \times \mathrm{LD}_{50}$ via inhalational challenge (58). The injectable vaccine also failed to adequately protect African green monkeys against aerosolized Y. pestis (19). Other reports have shown that $\mathrm{rF1}-\mathrm{LcrV}$ immunization through the non-invasive i.n. route conferred $80-90 \%$ protection against 70 to $100 \times \mathrm{LD}_{50}$ of $Y$. pestis via inhalational challenge $(58,59)$. Finally, Jones et al. (32) demonstrated that immunization with ProtollinF1-V through an i.n. route elicits $80 \%$ protection against an aerosol challenge at $255 \times \mathrm{LD}_{50}$ of $Y$. pestis and $100 \%$ protection against $170 \times \mathrm{LD}_{50}$ of $Y$. pestis. Overall, these studies indicate that the route of inoculation affects vaccine effectiveness against pneumonic plague. Modifying the antigen, we created the rF1V10 fusion protein and established a mouse model of i.t. delivery to investigate protective immunity against pneumonic plague. Our results demonstrate that i.t.-rF1-V10 immunization induces strong immune responses that lead to $100 \%$ protection against an aerosol challenge of high-dose $Y$. pestis strain 201. Moreover, i.t. inoculation is an improved non-invasive method for pulmonary delivery of vaccines in mice compared to conventional i.n. inoculation that has several disadvantages: a low residence time; mucociliary clearance of vaccines in the nose, throat and upper airways leads to an inefficient uptake of soluble antigens; the route is unsuitable for powder pulmonary delivery; and it is not possible to quantify the given dose (60-62). We propose that the i.t. immunization with inhalable $\mathrm{rF} 1-\mathrm{V} 10$ vaccine may provide an alternative, possibly better, vaccination strategy against pneumonic plague.

Compared to pulmonary delivery (i.n., i.t. etc.) of liquid vaccine, powder formulation delivered by i.t. route is more advantageous (63). The F1-V SFD powders reconstituted in water delivered by i.n. route provided at most $80 \%$ protection against bubonic plague, however, the immunization of powder formulation was not included in this previous study (64). We recently demonstrated the liquid formulation of EV76-BSHU $\Delta$ pla delivered by i.t. route represents an excellent liveattenuated vaccine candidate against pneumonic plague (54), but it is likely unsuitable for preparation of powder by SFD, which will affect survivability and overall fitness of the live bacteria. In the current study, we evaluated the protection efficacy of three subunit vaccines administrated with three different formulations via i.t. route. Our results demonstrate that protection against an aerosolized $Y$. pestis challenge conferred by i.t. delivery of vaccines in dry powder formulations is at least equivalent to that conferred by i.t. delivery of liquid formulations of the same vaccine. Potential advantages of dry powder over liquid vaccines include: (i) the powder formulations could eliminate the coldchain requirement, thus considerably reducing the costs of storage and shipping (60); (ii) the improved antigen stability may enhance the immunity induced after vaccination (65); (iii) using excipients as bulking agent could increase the total amount

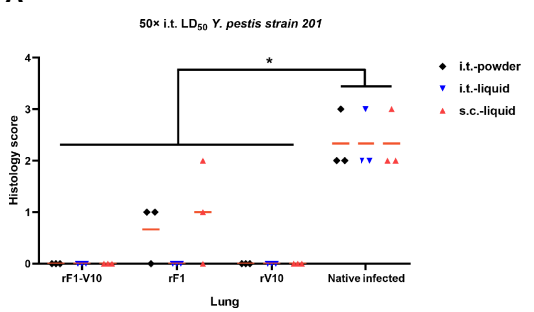

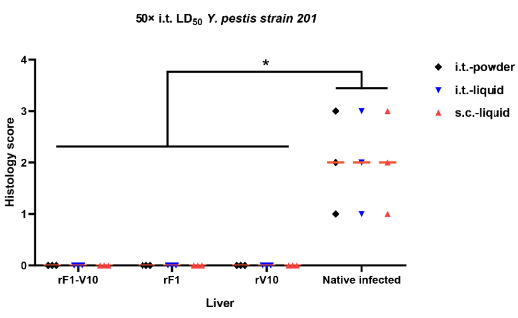

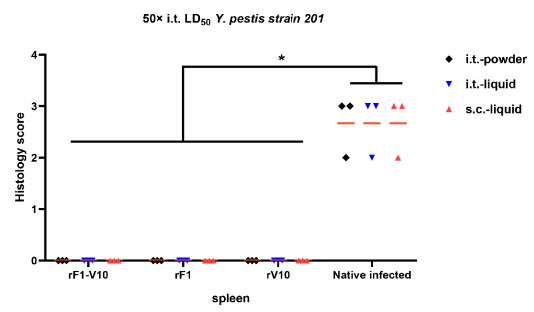

FIGURE 7 | Pathological lesions in the tissues of mice euthanized at 2 days post $50 \times L_{50}$ Y. pestis strain 201 i.t. challenge. Tissue from lungs, livers, and spleens were collected from three mice per group ( $\mathrm{rF1}$-V10, $\mathrm{rF1}$, and rV10 with different formulations via i.t. or s.c. routes), fixed in formalin, embedded in paraffin, and stained with HE. Pathological scores of (A) lung tissue; (B) liver tissue; and (C) spleen tissue are presented. Tissue sections were evaluated by a trained pathologist according to the following scores: 0 , no pathological lesions; 1 , minimal; 2, mild; 3 , moderate; 4 , severe. The degree of pathological lesions was related to the distribution and severity of lesions as follows: (I) edema; (II) tissue parenchymatous lesions, such as congestion and hemorrhage. Experiments were performed twice independently with similar results. Data are expressed as the mean $\pm \mathrm{SD}(\mathrm{n}=3)$ for data collected from one representative experiment. ${ }^{\star} P<0.05$. 
of inhaled powders, which makes given dose more accurate (66). These advantages suggest that powder formulation immunization via i.t. can provide a promising improvement over the existing vaccine.

At the mucosal surfaces, the predominant immunoglobulin is secretory IgA (SIgA). SIgA-based protective mucosal immunity can prevent an infectious agent from entering the body and block microbial toxins from binding to, or affecting, epithelial and other target cells (67). Some studies suggest that specific SIgA plays a key role in neutralizing pathogens or toxins $(68,69)$. One limitation of this study is that we didn't explain why s.c.-rF1-V10 vaccination conferred complete protection against a high dose $Y$. pestis challenge without inducing SIgA production. This result indicated that the role of specific SIgA in protecting against respiratory infection of $Y$. pestis needs to be further investigated, such as evaluating the survival rate of mice challenged with $Y$. pestis that had been preincubated with SIgA (70), or evaluating the efficacy of subunit vaccines via i.t. delivery in wild-type (WT) and $\operatorname{IgA-deficient~(IgA~(-/-))~mice~(71).~Another~limitation~is~}$ that we didn't evaluate the innate immune response in the early stage of i.t. immunization, which is supposed to be more efficient than that of s.c. immunization $(72,73)$.

Taken together, we have demonstrated that the rF1-V10 fusion protein vaccine can confer complete protection against a high dose aerosolized $Y$. pestis challenge and that i.t. delivery of vaccines can induce higher protection efficacy in mice compared to that of s.c. immunization. However, i.t. route might be only suitable for animal use. For human pulmonary delivery of dry powder vaccine, the widely accepted inhaled devices named dry powder inhalers (DPIs) are recommended, which are easy to administrate without the assistant of trained medical personnel $(65,74)$. The use of this alternative method of pulmonary delivery and powder vaccine formulations may directly benefit biodefense vaccination programs and, ultimately, facilitate mass vaccination.

\section{REFERENCES}

1. Feodorova VA, Corbel MJ. Prospects for New Plague Vaccines. Expert Rev Vaccines (2009) 8(12):1721-38. doi: 10.1586/erv.09.129

2. Drancourt M, Raoult D. Molecular History of Plague. Clin Microbiol Infect (2016) 22(11):911-5. doi: 10.1016/j.cmi.2016.08.031

3. Ke Y, Chen Z, Yang R. Yersinia Pestis: Mechanisms of Entry Into and Resistance to the Host Cell. Front Cell Infect Microbiol (2013) 3:106. doi: $10.3389 /$ fcimb.2013.00106

4. Verma SK, Tuteja U. Plague Vaccine Development: Current Research and Future Trends. Front Immunol (2016) 7:602. doi: 10.3389/fimmu. 2016.00602

5. Barbieri R, Signoli M, Chevé D, Costedoat C, Tzortzis S, Aboudharam G, et al. Yersinia Pestis: The Natural History of Plague. Clin Microbiol Rev (2020) 34 (1):e0044-19. doi: 10.1128/cmr.00044-19

6. Perry RD, Fetherston JD. Yersinia Pestis-Etiologic Agent of Plague. Clin Microbiol Rev (1997) 10(1):35-66. doi: 10.1128/cmr.10.1.35

7. Yang R. Plague: Recognition, Treatment, and Prevention. J Clin Microbiol (2018) 56(1):e01519-17. doi: 10.1128/jcm.01519-17

8. Sebbane F, Jarrett CO, Gardner D, Long D, Hinnebusch BJ. Role of the Yersinia Pestis Plasminogen Activator in the Incidence of Distinct Septicemic and Bubonic Forms of Flea-Borne Plague. Proc Natl Acad Sci U S A (2006) 103 (14):5526-30. doi: 10.1073/pnas.0509544103

\section{DATA AVAILABILITY STATEMENT}

The datasets presented in this study can be found in online repositories. The names of the repository/repositories and accession number(s) can be found in the article/Supplementary Material.

\section{ETHICS STATEMENT}

The animal study was reviewed and approved by the Institute of Animal Care and Use Committee (IACUC) at the Academy of Military Medical Sciences (AMMS).

\section{AUTHOR CONTRIBUTIONS}

$\mathrm{DZ}, \mathrm{XX}$, and WY designed the experiments. WZ, XS, LLZ, and LNZ performed animal experiments. ML and LH finished other experiments together. JG and $\mathrm{XZ}$ analyzed data and drew the figures. WZ, XS, DZ, XX, and WY revised the manuscript. All authors approved the article.

\section{ACKNOWLEDGMENTS}

The authors thank Yanchun Wang and Jianing Zhang for technical assistance in this study. We also thank MedPeer (www.medpeer.cn) for its linguistic assistance during the preparation of this manuscript.

\section{SUPPLEMENTARY MATERIAL}

The Supplementary Material for this article can be found online at: https://www.frontiersin.org/articles/10.3389/fimmu.2022. 793382/full\#supplementary-material

9. Pechous RD, Sivaraman V, Stasulli NM, Goldman WE. Pneumonic Plague: The Darker Side of Yersinia Pestis. Trends Microbiol (2016) 24(3):190-7. doi: 10.1016/j.tim.2015.11.008

10. Prentice MB, Rahalison L. Plague. Lancet (2007) 369(9568):1196-207. doi: 10.1016/s0140-6736(07)60566-2

11. Inglesby TV, Dennis DT, Henderson DA, Bartlett JG, Ascher MS, Eitzen E, et al. Plague as a Biological Weapon: Medical and Public Health Management. Working Group on Civilian Biodefense. Jama (2000) 283(17):2281-90. doi: 10.1001/jama.283.17.2281

12. Meyer KF. Effectiveness of Live or Killed Plague Vaccines in Man. Bull World Health Organ (1970) 42(5):653-66.

13. Russell P, Eley SM, Hibbs SE, Manchee RJ, Stagg AJ, Titball RW. A Comparison of Plague Vaccine, USP and EV76 Vaccine Induced Protection Against Yersinia Pestis in a Murine Model. Vaccine (1995) 13(16):1551-6. doi: $10.1016 / 0264-410 x(95) 00090-n$

14. Cohen RJ, Stockard JL. Pneumonic Plague in an Untreated Plague-Vaccinated Individual. Jama (1967) 202(4):365-6. doi: 10.1001/jama.1967.0313017 0165036

15. Titball RW, Williamson ED. Yersinia Pestis (Plague) Vaccines. Expert Opin Biol Ther (2004) 4(6):965-73. doi: 10.1517/14712598.4.6.965

16. Girard G. [Immunity in Plague. Acquisitions Supplied by 30 Years of Work on The "Pasteurella Pestis Ev" (Girard and Robic) Strain]. Biol Med (1963) 52:631-731. L'immunit'e dans l'infection pesteuse. Acquisitions apport'ees 
par 30 ann'ees de travaux sur la souche de "pasteurella pestis ev" (girard et robic). fre.

17. Wang X, Zhang X, Zhou D, Yang R. Live-Attenuated Yersinia Pestis Vaccines. Expert Rev Vaccines (2013) 12(6):677-86. doi: 10.1586/erv.13.42

18. Feodorova VA, Sayapina LV, Corbel MJ, Motin VL. Russian Vaccines Against Especially Dangerous Bacterial Pathogens. Emerg Microbes Infect (2014) 3 (12):e86. doi: $10.1038 / \mathrm{emi} .2014 .82$

19. Smiley ST. Current Challenges in the Development of Vaccines for Pneumonic Plague. Expert Rev Vaccines (2008) 7(2):209-21. doi: 10.1586/ 14760584.7.2.209

20. Baker EE, Sommer H, Foster LE, Meyer E, Meyer KF. Studies on Immunization Against Plague. I. The Isolation and Characterization of the Soluble Antigen of Pasteurella Pestis. J Immunol (1952) 68(2):131-45.

21. Andrews GP, Heath DG, Anderson GW Jr, Welkos SL, Friedlander AM. Fraction 1 Capsular Antigen (F1) Purification From Yersinia Pestis CO92 and From an Escherichia Coli Recombinant Strain and Efficacy Against Lethal Plague Challenge. Infect Immun (1996) 64(6):2180-7. doi: 10.1128/ iai.64.6.2180-2187.1996

22. Sha J, Endsley JJ, Kirtley ML, Foltz SM, Huante MB, Erova TE, et al. Characterization of an F1 Deletion Mutant of Yersinia Pestis CO92, Pathogenic Role of F1 Antigen in Bubonic and Pneumonic Plague, and Evaluation of Sensitivity and Specificity of F1 Antigen Capture-Based Dipsticks. J Clin Microbiol (2011) 49(5):1708-15. doi: 10.1128/jcm.00064-11

23. Burrows TW. An Antigen Determining Virulence in Pasteurella Pestis. Nature (1956) 177(4505):426-7. doi: 10.1038/177426b0

24. Anderson GW Jr, Leary SE, Williamson ED, Titball RW, Welkos SL, Worsham PL, et al. Recombinant V Antigen Protects Mice Against Pneumonic and Bubonic Plague Caused by F1-Capsule-Positive and -Negative Strains of Yersinia Pestis. Infect Immun (1996) 64(11):4580-5. doi: 10.1128/iai.64.11.4580-4585.1996

25. Batra L, Verma SK, Nagar DP, Saxena N, Pathak P, Pant SC, et al. HSP70 Domain II of Mycobacterium Tuberculosis Modulates Immune Response and Protective Potential of F1 and LcrV Antigens of Yersinia Pestis in a Mouse Model. PloS Negl Trop Dis (2014) 8(12):e3322. doi: 10.1371/journal.pntd. 0003322

26. Nakajima R, Motin VL, Brubaker RR. Suppression of Cytokines in Mice by Protein A-V Antigen Fusion Peptide and Restoration of Synthesis by Active Immunization. Infect Immun (1995) 63(8):3021-9. doi: 10.1128/iai.63.8.30213029.1995

27. Overheim KA, Depaolo RW, Debord KL, Morrin EM, Anderson DM, Green NM, et al. LcrV Plague Vaccine With Altered Immunomodulatory Properties. Infect Immun (2005) 73(8):5152-9. doi: 10.1128/iai.73.8.5152-5159.2005

28. Liu L, Wei D, Qu Z, Sun L, Miao Y, Yang Y, et al. A Safety and Immunogenicity Study of a Novel Subunit Plague Vaccine in Cynomolgus Macaques. J Appl Toxicol (2018) 38(3):408-17. doi: 10.1002/jat.3550

29. Qi Z, Zhou L, Zhang Q, Ren L, Dai R, Wu B, et al. Comparison of Mouse, Guinea Pig and Rabbit Models for Evaluation of Plague Subunit Vaccine F1 +Rv270. Vaccine (2010) 28(6):1655-60. doi: 10.1016/j.vaccine.2009.02.078

30. Williamson ED, Eley SM, Griffin KF, Green M, Russell P, Leary SE, et al. A New Improved Sub-Unit Vaccine for Plague: The Basis of Protection. FEMS Immunol Med Microbiol (1995) 12(3-4):223-30. doi: 10.1111/j.1574695X.1995.tb00196.x

31. Williamson ED, Sharp GJ, Eley SM, Vesey PM, Pepper TC, Titball RW, et al. Local and Systemic Immune Response to a Microencapsulated Sub-Unit Vaccine for Plague. Vaccine (1996) 14(17-18):1613-9. doi: 10.1016/s0264410x(96)00151-x

32. Jones T, Adamovicz JJ, Cyr SL, Bolt CR, Bellerose N, Pitt LM, et al. Intranasal Protollin/F1-V Vaccine Elicits Respiratory and Serum Antibody Responses and Protects Mice Against Lethal Aerosolized Plague Infection. Vaccine (2006) 24(10):1625-32. doi: 10.1016/j.vaccine.2005.09.052

33. Erova TE, Rosenzweig JA, Sha J, Suarez G, Sierra JC, Kirtley ML, et al. Evaluation of Protective Potential of Yersinia Pestis Outer Membrane Protein Antigens as Possible Candidates for a New-Generation Recombinant Plague Vaccine. Clin Vaccine Immunol (2013) 20(2):227-38. doi: 10.1128/ cvi.00597-12

34. Bowen W, Batra L, Pulsifer AR, Yolcu ES, Lawrenz MB, Shirwan H. Robust Th1 Cellular and Humoral Responses Generated by the Yersinia Pestis Rf1-V Subunit Vaccine Formulated to Contain an Agonist of the CD137 Pathway do
Not Translate Into Increased Protection Against Pneumonic Plague. Vaccine (2019) 37(38):5708-16. doi: 10.1016/j.vaccine.2019.07.103

35. Feodorova VA, Motin VL. Plague Vaccines: Current Developments and Future Perspectives. Emerg Microbes Infect (2012) 1(11):e36. doi: 10.1038/ emi.2012.34

36. Lyadova IV, Vordermeier HM, Eruslanov EB, Khaidukov SV, Apt AS, Hewinson RG. Intranasal BCG Vaccination Protects BALB/c Mice Against Virulent Mycobacterium Bovis and Accelerates Production of IFN-Gamma in Their Lungs. Clin Exp Immunol (2001) 126(2):274-9. doi: 10.1046/j.13652249.2001.01667.x

37. Garcia-Contreras L, Wong YL, Muttil P, Padilla D, Sadoff J, Derousse J, et al. Immunization by a Bacterial Aerosol. Proc Natl Acad Sci U S A (2008) 105 (12):4656-60. doi: 10.1073/pnas.0800043105

38. Lycke N. Recent Progress in Mucosal Vaccine Development: Potential and Limitations. Nat Rev Immunol (2012) 12(8):592-605. doi: 10.1038/nri3251

39. Patton JS, Byron PR. Inhaling Medicines: Delivering Drugs to the Body Through the Lungs. Nat Rev Drug Discov (2007) 6(1):67-74. doi: 10.1038/ $\operatorname{nrd} 2153$

40. Bramwell VW, Eyles JE, Oya Alpar H. Particulate Delivery Systems for Biodefense Subunit Vaccines. Adv Drug Deliv Rev (2005) 57(9):1247-65. doi: 10.1016/j.addr.2005.01.010

41. Illum L, Jabbal-Gill I, Hinchcliffe M, Fisher AN, Davis SS. Chitosan as a Novel Nasal Delivery System for Vaccines. Adv Drug Deliv Rev (2001) 51(1-3):8196. doi: 10.1016/s0169-409x(01)00171-5

42. Garmise RJ, Staats HF, Hickey AJ. Novel Dry Powder Preparations of Whole Inactivated Influenza Virus for Nasal Vaccination. AAPS PharmSciTech (2007) 8(4):E81. doi: 10.1208/pt0804081

43. Coulanges P. [50th Anniversary of the EV Antiplague Vaccine (Girard and Robic)]. Bull Soc Pathol Exot Filiales (1983) 76(2):114-20. Cinquantenaire du vaccin antipesteux EV (Girard et Robic). fre.

44. Coulanges P. [The 50th Anniversary of the Anti-Plague Vaccine EV (Girard and Robic)]. Arch Inst Pasteur Madagascar (1982) 50(1):169-84. Cinquantenaire du vaccin antipesteux EV (Girard et Robic). fre.

45. Meyer KF, Smith G, Foster L, Brookman M, Sung M. Live, Attenuated Yersinia Pestis Vaccine: Virulent in Nonhuman Primates, Harmless to Guinea Pigs. J Infect Dis (1974) 129:Suppl:S85-12. doi: 10.1093/infdis/ 129.supplement_1.s85

46. Powell BS, Andrews GP, Enama JT, Jendrek S, Bolt C, Worsham P, et al. Design and Testing for a Nontagged F1-V Fusion Protein as Vaccine Antigen Against Bubonic and Pneumonic Plague. Biotechnol Prog (2005) 21(5):1490510. doi: $10.1021 / \mathrm{bp} 050098 \mathrm{r}$

47. Sing A, Rost D, Tvardovskaia N, Roggenkamp A, Wiedemann A, Kirschning CJ, et al. Yersinia V-Antigen Exploits Toll-Like Receptor 2 and CD14 for Interleukin 10-Mediated Immunosuppression. J Exp Med (2002) 196(8):101724. doi: $10.1084 /$ jem. 20020908

48. Sawa T, Yahr TL, Ohara M, Kurahashi K, Gropper MA, Wiener-Kronish JP, et al. Active and Passive Immunization With the Pseudomonas V Antigen Protects Against Type III Intoxication and Lung Injury. Nat Med (1999) 5 (4):392-8. doi: 10.1038/7391

49. Torge A, Grützmacher P, Mücklich F, Schneider M. The Influence of Mannitol on Morphology and Disintegration of Spray-Dried NanoEmbedded Microparticles. Eur J Pharm Sci (2017) 104:171-9. doi: 10.1016/ j.ejps.2017.04.003

50. Gan C, Luo W, Yu Y, Jiao Z, Li S, Su D, et al. Intratracheal Inoculation of AHc Vaccine Induces Protection Against Aerosolized Botulinum Neurotoxin A Challenge in Mice. NPJ Vaccines (2021) 6(1):87. doi: 10.1038/s41541-02100349-w

51. Fukuyama Y, Ikeda Y, Ohori J, Sugita G, Aso K, Fujihashi K, et al. A Molecular Mucosal Adjuvant to Enhance Immunity Against Pneumococcal Infection in the Elderly. Immune Netw (2015) 15(1):9-15. doi: 10.4110/in.2015.15.1.9

52. Harris JA, Stein SW, Myrdal PB. Evaluation of the TSI Aerosol Impactor 3306/3321 System Using a Redesigned Impactor Stage With Solution and Suspension Metered-Dose Inhalers. AAPS PharmSciTech (2006) 7(1):E138e145. doi: $10.1208 / \mathrm{pt} 070120$

53. Tian G, Qi Z, Qiu Y, Wu X, Zhang Q, Yang X, et al. Comparison of Virulence Between the Yersinia Pestis Microtus 201, an Avirulent Strain to Humans, and the Vaccine Strain EV in Rhesus Macaques, Macaca Mulatta. Hum Vaccin Immunother (2014) 10(12):3552-60. doi: 10.4161/hv.35119 
54. Feng J, Deng Y, Fu M, Hu X, Luo W, Lu Z, et al. Construction of a LiveAttenuated Vaccine Strain of Yersinia Pestis EV76-B-Shuspla and Evaluation of Its Protection Efficacy in a Mouse Model by Aerosolized Intratracheal Inoculation. Front Cell Infect Microbiol (2020) 10:473. doi: 10.3389/ fcimb.2020.00473

55. Feng J, Hu X, Fu M, Dai L, Yu Y, Luo W, et al. Enhanced Protection Against Q Fever in BALB/c Mice Elicited by Immunization of Chloroform-Methanol Residue of Coxiella Burnetii via Intratracheal Inoculation. Vaccine (2019) 37 (41):6076-84. doi: 10.1016/j.vaccine.2019.08.041

56. Xiong X, Qi Y, Jiao J, Gong W, Duan C, Wen B. Exploratory Study on Th1 Epitope-Induced Protective Immunity Against Coxiella Burnetii Infection. PloS One (2014) 9(1):e87206. doi: 10.1371/journal.pone.0087206

57. Chen K, Magri G, Grasset EK, Cerutti A. Rethinking Mucosal Antibody Responses: IgM, IgG and IgD Join IgA. Nat Rev Immunol (2020) 20(7):42741. doi: 10.1038/s41577-019-0261-1

58. Glynn A, Roy CJ, Powell BS, Adamovicz JJ, Freytag LC, Clements JD. Protection Against Aerosolized Yersinia Pestis Challenge Following Homologous and Heterologous Prime-Boost With Recombinant Plague Antigens. Infect Immun (2005) 73(8):5256-61. doi: 10.1128/iai.73.8.52565261.2005

59. Yamanaka H, Hoyt T, Yang X, Golden S, Bosio CM, Crist K, et al. A Nasal Interleukin-12 DNA Vaccine Coexpressing Yersinia Pestis F1-V Fusion Protein Confers Protection Against Pneumonic Plague. Infect Immun (2008) 76(10):4564-73. doi: 10.1128/iai.00581-08

60. Amorij JP, Saluja V, Petersen AH, Hinrichs WL, Huckriede A, Frijlink HW. Pulmonary Delivery of an Inulin-Stabilized Influenza Subunit Vaccine Prepared by Spray-Freeze Drying Induces Systemic, Mucosal Humoral as Well as Cell-Mediated Immune Responses in BALB/c Mice. Vaccine (2007) 25 (52):8707-17. doi: 10.1016/j.vaccine.2007.10.035

61. Jabbal-Gill I. Nasal Vaccine Innovation. J Drug Target (2010) 18(10):771-86. doi: 10.3109/1061186x.2010.523790

62. Tomar J, Born PA, Frijlink HW, Hinrichs WL. Dry Influenza Vaccines: Towards a Stable, Effective and Convenient Alternative to Conventional Parenteral Influenza Vaccination. Expert Rev Vaccines (2016) 15(11):143147. doi: 10.1080/14760584.2016.1182869

63. Beck SE, Laube BL, Barberena CI, Fischer AC, Adams RJ, Chesnut K, et al. Deposition and Expression of Aerosolized rAAV Vectors in the Lungs of Rhesus Macaques. Mol Ther J Am Soc Gene Ther (2002) 6(4):546-54. doi: $10.1006 / \mathrm{mthe} .2002 .0698$

64. Huang J, D'Souza AJ, Alarcon JB, Mikszta JA, Ford BM, Ferriter MS, et al. Protective Immunity in Mice Achieved With Dry Powder Formulation and Alternative Delivery of Plague F1-V Vaccine. Clin Vaccine Immunol (2009) 16 (5):719-25. doi: 10.1128/cvi.00447-08

65. Sou T, Meeusen EN, de Veer M, Morton DA, Kaminskas LM, McIntosh MP. New Developments in Dry Powder Pulmonary Vaccine Delivery. Trends Biotechnol (2011) 29(4):191-8. doi: 10.1016/j.tibtech.2010.12.009
66. Tonnis WF, Lexmond AJ, Frijlink HW, de Boer AH, Hinrichs WL. Devices and Formulations for Pulmonary Vaccination. Expert Opin Drug Deliv (2013) 10(10):1383-97. doi: 10.1517/17425247.2013.810622

67. Mantis NJ, Rol N, Corthésy B. Secretory IgA's Complex Roles in Immunity and Mucosal Homeostasis in the Gut. Mucosal Immunol (2011) 4(6):603-11. doi: $10.1038 / \mathrm{mi} .2011 .41$

68. Holmgren J, Czerkinsky C. Mucosal Immunity and Vaccines. Nat Med (2005) 11(4 Suppl):S45-53. doi: 10.1038/nm1213

69. Mantis NJ, McGuinness CR, Sonuyi O, Edwards G, Farrant SA. Immunoglobulin A Antibodies Against Ricin A and B Subunits Protect Epithelial Cells From Ricin Intoxication. Infect Immun (2006) 74(6):345562. doi: 10.1128/iai.02088-05

70. Kobayashi R, Kohda T, Kataoka K, Ihara H, Kozaki S, Pascual DW, et al. A Novel Neurotoxoid Vaccine Prevents Mucosal Botulism. J Immunol (2005) 174(4):2190-5. doi: 10.4049/jimmunol.174.4.2190

71. Rodríguez A, Tjärnlund A, Ivanji J, Singh M, García I, Williams A, et al. Role of IgA in the Defense Against Respiratory Infections IgA Deficient Mice Exhibited Increased Susceptibility to Intranasal Infection With Mycobacterium Bovis BCG. Vaccine (2005) 23(20):2565-72. doi: 10.1016/ j.vaccine.2004.11.032

72. Piggott DA, Eisenbarth SC, Xu L, Constant SL, Huleatt JW, Herrick CA, et al. MyD88-Dependent Induction of Allergic Th2 Responses to Intranasal Antigen. J Clin Invest (2005) 115(2):459-67. doi: 10.1172/jci22462

73. Neutra MR, Kozlowski PA. Mucosal Vaccines: The Promise and the Challenge. Nat Rev Immunol (2006) 6(2):148-58. doi: 10.1038/nri1777

74. Dal Negro RW. Dry Powder Inhalers and the Right Things to Remember: A Concept Review. Multidiscip Respir Med (2015) 10(1):13. doi: 10.1186/ s40248-015-0012-5

Conflict of Interest: The authors declare that the research was conducted in the absence of any commercial or financial relationships that could be construed as a potential conflict of interest.

Publisher's Note: All claims expressed in this article are solely those of the authors and do not necessarily represent those of their affiliated organizations, or those of the publisher, the editors and the reviewers. Any product that may be evaluated in this article, or claim that may be made by its manufacturer, is not guaranteed or endorsed by the publisher.

Copyright (C) 2022 Zhang, Song, Zhai, Guo, Zheng, Zhang, Lv, Hu, Zhou, Xiong and Yang. This is an open-access article distributed under the terms of the Creative Commons Attribution License (CC BY). The use, distribution or reproduction in other forums is permitted, provided the original author(s) and the copyright owner(s) are credited and that the original publication in this journal is cited, in accordance with accepted academic practice. No use, distribution or reproduction is permitted which does not comply with these terms. 